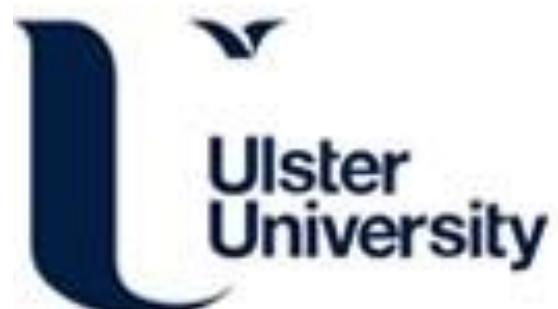

\section{Decoding of wrist and finger movement from electroencephalography signal}

Pal, M., Bhattacharyya, S., Konar, A., Tibarewala, D. N., \& Janarthanan, R. (2014). Decoding of wrist and finger movement from electroencephalography signal. In IEEE CONECCT 2014 - 2014 IEEE International Conference on Electronics, Computing and Communication Technologies [6740323] (IEEE CONECCT 2014 - 2014 IEEE International Conference on Electronics, Computing and Communication Technologies). IEEE Computer Society. https://doi.org/10.1109/CONECCT.2014.6740323

Link to publication record in Ulster University Research Portal

Published in:

IEEE CONECCT 2014 - 2014 IEEE International Conference on Electronics, Computing and Communication Technologies

Publication Status:

Published (in print/issue): 01/01/2014

DOI:

10.1109/CONECCT.2014.6740323

\section{Document Version}

Author Accepted version

\section{General rights}

Copyright for the publications made accessible via Ulster University's Research Portal is retained by the author(s) and / or other copyright owners and it is a condition of accessing these publications that users recognise and abide by the legal requirements associated with these rights.

\section{Take down policy}

The Research Portal is Ulster University's institutional repository that provides access to Ulster's research outputs. Every effort has been made to ensure that content in the Research Portal does not infringe any person's rights, or applicable UK laws. If you discover content in the Research Portal that you believe breaches copyright or violates any law, please contact pure-support@ulster.ac.uk. 


\title{
Decoding of Wrist and Finger Movement from Electroencephalography Signal
}

\author{
Monalisa Pal $^{1, \mathrm{a}}$, Saugat Bhattacharyya ${ }^{1, \mathrm{~b}}$, Amit Konar ${ }^{1, \mathrm{c}}$, D.N. Tibarewala ${ }^{2, \mathrm{~d}}$, R. Janarthanan ${ }^{3, \mathrm{e}}$ \\ ${ }^{1}$ Dept. of Electronics \&Telecommunication Engg, ${ }^{2}$ School of Bioscience \& Engg, ${ }^{3}$ Dept. of Computer Science. \\ ${ }^{1,2}$ Jadavpur University, ${ }^{3}$ TJS Engineering College. \\ ${ }^{1,2}$ Kolkata, ${ }^{3}$ Chennai, India. \\ amonalisap90@gmail.com, bsaugatbhattacharyya@live.com, ${ }^{c}$ konaramit@yahoo.co.in, dbiomed.ju@gmail.com, \\ esrmjana_73@yahoo.com.
}

\begin{abstract}
The emergence of brain-computer interfacing has made the control of robots through thought a reality. Such realtime application calls for fast processing and accurate classification of brain signals. In this paper, we address the twolevel classification of motor imagery signals, where the user differentiates between clockwise/ counter-clockwise movement of wrist and the opening/closing of the fingers. For this purpose, parameters of adaptive autoregressive (AAR) models and Extreme Energy Ratio criterion (EER) are employed as features, which are fed to standard classifiers for comparison. It concludes the features extracted based on EER, selected by sequential forward search and classified using radial basis function kernelized support vector machine, provides optimum performance of the classification process for implementation in real-time scenario, with an average accuracy $90.24 \%$ and a time complexity of 8.2449 seconds.
\end{abstract}

Index Terms-Adaptive Auto-Regressive Model, BrainComputer Interfacing, Distance Likelihood Ratio Test, Electroencephalography, Extreme Energy Ratio, Fisher Linear Discriminant, Naïve Bayes, Radial Basis Function based Support Vector Machine, Sequential Forward Feature Selection.

\section{INTRODUCTION}

Brain-Computer Interfacing (BCI) deals with decoding the mental states of the human brain which can be used as a control interface to any external computing device. The main steps of $\mathrm{BCI}$ includes acquisition of signals representing brain activities, processing the acquired signal to extract the relevant features, classification of the extracted features, controlling an external device using the classification output and obtaining a feedback of the response from the subject [1-2]. One of the major practical applications of $\mathrm{BCI}$ is building a non-muscular communication channel between the brain of a mobilitydisabled person and a prosthetic device bypassing the human nerves and muscles. The spectrum of areas in which BCI is useful includes robotics, military services, virtual gaming controls, mass communication, healthcare, navigation, etc [34].

There are several ways that measure the neuronal firing inside the brain. A few of these methods include functional magnetic resonance imaging (fMRI), magnetic encephalography (MEG), functional near-infrared spectroscopy
(fNIRS) electro-corticography (ECoG), intra-cortical electrodes and electroencephalography (EEG) [5]. EEG is the preferred device for real time application in BCI because it provides high temporal resolution, non-invasive, easily available and portable [6].

Previous researches on EEG-based BCI have successfully discriminated among the left-right movement imagery signals using various signal processing and classification techniques. In [7-8], the researchers have claimed that during movement imagination or execution, event related synchronization (ERS) occurs in the $\gamma$ band and event related desynchronization (ERD) is found to be prevalent in the $\mu$ and $\beta$ bands. These signals originate from the somatosensory and motor cortex region of the brain. In [9-14], EEG based BCI has been used in decoding of limb movements. Such works has been has been successfully implemented on neuro-prosthetic devices [1]. In [13], researchers have demonstrated the use of 2-fold classification using RBF-SVM.

In this study, we aim to discriminate among clockwise and counter-clockwise movement of the wrist, and opening and closing of the fingers. For this purpose, we have employed the parameters of adaptive auto-regressive models and extreme energy ratio criterion as features. Following feature extraction, sequential forward feature selection is used as a feature reduction technique to remove the redundant features from the original feature vector. The reduced feature vector is then fed to the classifiers: Naïve Bayesian, Radial Basis Function kernelized Support Vector Machine, Fisher Linear Discriminant and Distance Likelihood Ratio Test to discriminate among the various mental states. In this work we aim at selecting the best feature-classifier pair that outperforms other pairs from the mentioned ones. This feature-classifier pair can be applied for future research work in this domain during real-time control of a prosthetic device.

Rest of the paper is organized as follows. Section II describes the theory and the methodology behind the entire work. Section III gives details on the experimental approach undertaken in this study with the results discussed in Section IV. Concluding remarks are given in Section V of this paper. 


\section{THEORETICAL BACKGROUND AND METHODOLOGY}

This section gives a brief description of the transformations used on the raw EEG data to obtain the required information of its corresponding mental states, highlighting the methods of feature extraction, feature selection and classification.

\section{A. Feature Extraction}

To represent the filtered EEG data in terms of characterizing attributes, we transform the data into distinguishable features. We consider two kinds of features to account for the non-linearity and non-stationarity of the EEG signal, which are,Adaptive Auto-Regressive (AAR) Parameters and Extreme Energy Ratio (EER) criterion.

\section{1) Adaptive Autoregressive Parameters}

Adaptive Auto-regressive model is similar to an autoregressive model but it takes into account the non-stationarity of a signal by varying the AR parameters in time, i.e., the AR parameters are estimated adaptively to get an AAR model. An adaptive auto-regressive model of order $p, A A R(p)$, is described by (1) and(2) where $x(n)$ is the $n$-th sample of the series under observation, $\eta(n)$ is the zero-mean-Gaussian noise with variance, $\sigma_{\eta}(n)^{2}$, and $a_{i}(n)$ are the time-varying AR coefficients. Any sample is predicted by past $p$ samples and the new information introduced by the noise. Thus, $\eta(n)$ is also called the innovation process. There are several algorithms that can be used for estimation of the AAR coefficients like least-meansquare (LMS) method, recursive-least-square (RLS) method, recursive AR (RAR) method, Kalman filtering, etc. [15-16].

$$
x(n)=\sum_{i=1}^{p} a_{i}(n) x(n-i)+\eta(n)
$$

with

$$
\eta(n)=N\left\{0, \sigma_{\eta}(n)^{2}\right\}
$$

In this paper, Kalman filtering is used as the estimation algorithm and the order selected for AAR modeling of the EEG signal is 6 as it yields optimum results. Accuracy decreases with a lower order model and does not improve if higher orders are used. The rate of adaptation of the AR coefficients is given by the update coefficient which is heuristically chosen to be 0.0085. This is small enough to allow the coefficients to change slowly. For the purpose of feature extraction, the 384 samples from a single electrode for an observation are fitted to an AAR(6) model thus, obtaining 6 coefficients for every sample. The other parameters required for Kalman filtering are learned during the adaptation. After the adaptation is complete, the 384 samples from each electrode are re-fitted to the model with newly learned Kalman filter parameters. This yields a $384 \times 6$ matrix from each sample which is further averaged to yield a $1 \times 6$ coefficient vector for each electrode.

\section{2) Extreme Energy Ratio}

EEG responses to a certain stimulus are assumed to be generated from some hidden signal sources beneath the surface of the brain cortex, which can be recovered by performing spatial filtering. At first, each of the EEG observations are rearranged such that we have a matrix $X$ of dimension $N \times T$ corresponding to a single trial. Here, $N$ is the number of electrodes and $T$ is the number of samples in the recording period. The sample covariance, $C$, when computed by (3) results in a matrix of $N \times N$ dimensions. The covariance of a particular class (say, $C_{0}$ and $C_{l}$ ) is obtained by averaging all the samples of that class. If $\phi$ is considered to be a spatial filter, the signal energy is given by $\phi^{T} X X^{T} \phi=\phi^{T} C \phi$. Then, the Extreme Energy Ratio (EER) criterion for distinguishing the classes is given by (4). There can be two filter $\phi_{\max }$ and $\phi_{\min }$ respectively for maximizing and minimizing the ratio in (4). The eigen vectors corresponding to the maximum and minimum eigen values of the matrix $C_{1}^{-1} C_{0}$ gives the spatial filters $\phi_{\max }$ and $\phi_{\min }$, respectively. The energy values of the signal filtered by these two filters can be treated as the features of an EEG observation. If $m$ sources are to be identified, we have $2 m$ values for each EEG sample. For $m$ sources, $\phi_{\max }\left(\phi_{\min }\right)$ is a set of filters given by $m$ generalized eigen vectors of matrix pair $\left(C_{0}, C_{l}\right)$ which correspond $m$ maximal (minimal) eigen values [17-18].

$$
\begin{gathered}
C=X X^{T} \\
R(\phi)=\frac{\phi^{T} C_{0} \phi}{\phi^{T} C_{1} \phi}
\end{gathered}
$$

In this paper, we consider 7 sources and correspondingly the feature vector for each sample is of the dimension $1 \times 14$. These are energy values of the filtered signals where the filters are the eigen vectors corresponding to the eigen values arranged in ascending order. So, the first (fourteenth) energy value in the feature vector is computed using the eigen vector corresponding to the smallest (largest) eigen value. Before feature extraction, each sample is re-arranged in $1 \times 384$ matrix for each electrode.

\section{B. Feature Selection: Sequential Forward Feature Selection}

Feature selection has been introduced to combat a few disadvantages of the classical machine learning algorithms. Often, the underlying function between input and output is determined not by all the features but by a subset of the extracted features. This subset should provide performance comparable to the complete feature set in lesser time. Thus, feature selection reduces computational cost.

In order to reduce the time complexity, people resort to greedy methods. One of such methods is sequential forward selection (SFS) [19-20]. In sequential forward selection, at first the single most relevant feature is selected. Following this, from the remaining features the best feature pairing with the chosen one is picked. Proceeding in a similar manner, the required subset is grown. For example, let us select a subset of $d$ features $\left\{f_{1}, f_{s_{2}}, \ldots, f_{s_{d}}\right\}$ from the available $D$ features, $\left\{f_{1}\right.$, $\left.f_{2}, \ldots, f_{\mathrm{D}}\right\}$ where $d \leq D$. At first, each of the $D$ features are tested the feature performing best is chosen as $f_{s_{l}}$. Each of the remaining $D-1$ features are paired with $f_{s_{1}}$ and evaluated. The pair performing best yields $\left\{f_{1}, f_{s_{2}}\right\}$. As we continue, the subset is developed till $d$ features are included. Let $f_{s_{l}}=f_{l}$. In the next step, we evaluate performance of $\left\{f_{1}, f_{2}\right\},\left\{f_{1}, f_{3}\right\}, \ldots$ $\left\{f_{1}, f_{D}\right\}$. But pairs like $\left\{f_{2}, f_{3}\right\}$ are not evaluated in this greedy process, which might have provided better performance as a pair than all the pairs with $f_{l}$. 
For the performance analysis of the chosen subset, we can use filter objective function or wrapper objective function. Any information-theoretic measure to assess the information content of the subset falls under the filter method whereas considering recognition rate of a pattern classifier on a test set formed on the subset of features is the wrapper method.

In this paper, from the set of features extracted, a subset of 10 features is selected using the wrapper method. Sequential forward feature selection has been exploited. The same classifier that evaluates the performance of the feature section algorithm has also been used for the purpose of classification.

\section{Classification}

We aim for a two-level classification, where at each level a binary classification is performed. Four supervised learning algorithms have been used which are briefly described here.

\section{1) Fisher Linear Discriminant}

The two classes $(y=0, y=1)$ must represent two compact and well-separated groups when projected in the feature space. Mathematically, compactness is indicated by small within-class covariance $\left(\sigma^{2}\right)$ of the features. We also note that two distant classes will have large difference between their mean $(\mu)$ features. Thus, in terms of statistics, Fisher Linear Discriminant (FLD) classifier [21] tries to build a hyperplane that maximizes the Fisher Discriminant Ratio (FDR) given by (5).

$$
S=\frac{\left(\mu_{y=0}-\mu_{y=1}\right)^{2}}{\sigma_{y=0}^{2}+\sigma_{y=1}^{2}}
$$

\section{2) Nä̈ve Bayesian}

A Bayesian classifier works on Bayes' theorem (6) from classical probability theory. However, it is a complex problem to find the composite distribution, $p\left(\underline{x} \mid C_{i}\right)$, of all the features given a particular class. In order to simplify this problem, the features are considered to be independent within a particular class (7). The Naïve Bayes classifier implements this independence in Bayes theorem to determine the class of any unknown feature vector $(8)$.

$$
\begin{gathered}
P\left(C_{i} \mid \underline{x}\right)=\frac{P\left(C_{i}\right) p\left(\underline{x} \mid C_{i}\right)}{\sum_{j=0}^{1} P\left(C_{j}\right) p\left(\underline{x} \mid C_{j}\right)} \\
p\left(\underline{x} \mid C_{i}\right)=p\left(x_{1}, x_{2}, \ldots x_{d} \mid C_{i}\right)=\prod_{j=1}^{d} p\left(x_{j} \mid C_{i}\right) \\
\text { Class }=\left\{\begin{array}{cc}
0 & \text { if } P\left(C_{0} \mid \underline{x}\right)>P\left(C_{1} \mid \underline{x}\right) \\
1 & \text { otherwise }
\end{array}\right.
\end{gathered}
$$

\section{3) Distance Likelihood Ratio Test}

We note that the denominator of Bayes' theorem (6) is same for all the classes, $C_{i}(i=0,1)$. As a consequence, the likelihood ratio, $R$, as given by (9) follows. When $R$ is more than 1 , decision favours class $C_{0}$ and otherwise the most likely class for the given feature is class $C_{l}$. Using some nonparametric estimation for the distribution, $R$ simplifies to a form given by (10) where $\Delta_{k}^{(i)}$ is the distance of the $k$-th neighbor in class $C_{i}, d$ is the dimensionality of the feature space and $n_{C i}$ is the fraction of sample of class $C_{i}$ within the considered neighbourhood. Thus, (10) gives the decision threshold for distance likelihood ratio test (DLRT) [22].

$$
\begin{gathered}
R=\frac{P\left(C_{0} \mid \underline{x}\right)}{P\left(C_{1} \mid \underline{x}\right)}=\frac{P\left(C_{0}\right) p\left(\underline{x} \mid C_{0}\right)}{P\left(C_{1}\right) p\left(\underline{x} \mid C_{1}\right)} \\
R=\frac{n_{C_{1}}}{n_{C_{0}}}\left(\frac{\Delta_{k^{(1)}}}{\Delta_{k^{(0)}}}\right)^{d}
\end{gathered}
$$

\section{4) Support Vector Machine}

Support Vector Machine (SVM) searches the best direction for the hyperplane i.e. the hyperplane with the largest margin that separates the data points of one class from those of the other classes. The data points belonging to either class that are closest to the separating hyperplane are called the support vectors. The hyperplane is specified only in terms of the support vectors. If the features are not linearly separable, they are projected into a different plane to obtain linearly separable classes. The projection is dictated by the use of kernels. The kernel function for the transformation can be a polynomial function, Gaussian function, radial basis function, sigmoid function, etc.

There has not been much of a choice in choosing the parameters for the FLD. However, for DLRT classifier a neighbourhood of 3 samples i.e., $\mathrm{k}=3$ and Euclidean distance are chosen as arguments. In case of Naïve Bayes classifier, the features are assumed to have multivariate normal distribution whose mean and covariance are learned during the process of training. A kernel employing the radial basis function has been used for the Support Vector Machine.

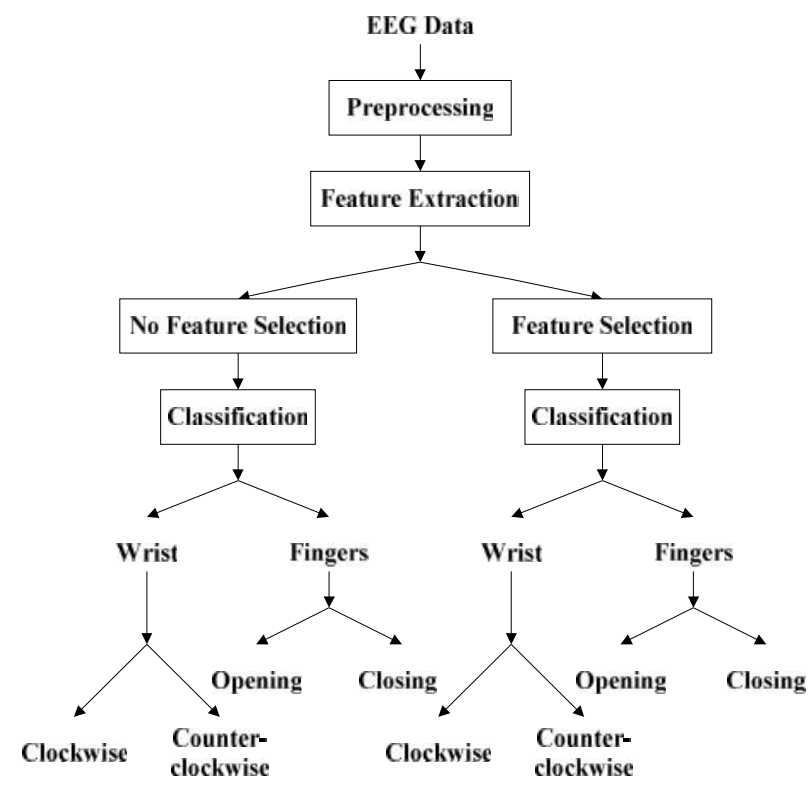

Fig. 1. Flowchart of the proposed scheme

The major steps of the proposed work have been outlined in the flowchart shown in Fig. 1. Features are extracted from the raw EEG data after being preprocessed. The set of features are then hierarchically classified after using feature selection as well as without feature selection. A two-level classification is done. The classes considered at level-1 are fingers and wrist 
which indicate the body part to which the movement is related. The sub-classes corresponding to the wrist class are clockwise and counter-clockwise which indicates the direction of movement. Similarly, the sub-classes associated with the fingers class are opening and closing analogous to grasping and releasing of any object.

\section{EXPERIMENTAL APPROACH}

The EEG data is collected from 8 subjects, 4 male and 4 female in the age group of $25 \pm 3$ years, for a period of 6 consecutive days. The EEG signal is acquired with the help of a 14 channel Emotiv headset which has a sampling rate $128 \mathrm{~Hz}$. The following electrodes are considered for acquisition: AF3, F7, F3, FC5, T7, P7, O1, O2, P8, T8, FC6, F4, F8 and AF4, all arranged according to the standard 10/20 system of electrode placement as shown in Fig. 2.

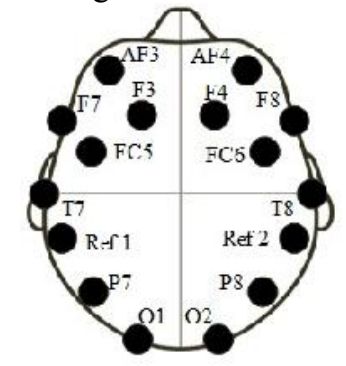

Fig. 2. EEG electrode placement

\section{A. Stimulus Generation}

The data acquisition consists of instructing the subjects through a sequence of visual stimulus or commands to perform the corresponding motor imagery movement, which is, clockwise/ counter-clockwise movement of the wrist and opening and closing of the fingers.

The generic structure of the visual stimuli is shown in Fig. 3. The blank command instructs the subject to relax and provides the baseline of the EEG. The ready command instructs the subject to be alert for the incoming command. For the opening and closing commands, the subject is instructed to grasp and release a ball kept near his/her right hand. When commands like clockwise and counter-clockwise appear, the subject is asked to rotate his/her right hand about the wrist in the azimuthal plane in the direction specified by the instruction. The ready, command and blank section is repeated 50 times for each of the four commands to obtain non-overlapping EEG responses.

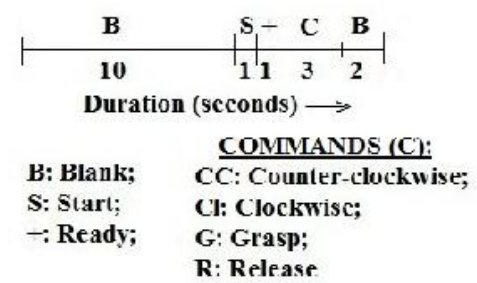

Fig. 3. Stimulus for EEG acquisition

\section{B. Preprocessing}

Based on modalities, the EEG signal can be grouped into different frequency bands. Informative motor imagery signals are picked up from $\mu(8-12 \mathrm{~Hz})$ and central $\beta(16-24 \mathrm{~Hz})$ band of EEG signals from the primary, supplementary and pre-motor cortex region of the brain.

Thus, we band-pass filter the raw (acquired) EEG signals in the bandwidth of range $8-25 \mathrm{~Hz}$ to remove other form of environmental and other cognitive noises from the signal. From experimentation, we have selected a $12^{\text {th }}$ order elliptical filter of $1 \mathrm{~dB}$ passband ripple and $50 \mathrm{~dB}$ stopband ripple to filter the raw EEG data. An elliptical filter is characterized by steeper roll-off characteristics and equiripple behavior in the passband and the stopband as compared to the other standard filters [23].

To nullify the effect of cross-talk from different and often adjacent electrodes, a spatial filtering method is required. Common average referencing is done on the raw data. As the name implies, it treats the mean voltage of the 14 electrodes as the reference and scales the whole data accordingly.

Based on the visual stimuli explained earlier, 3 seconds of the movement imagery data are considered for further processing from each trial. Following pre-processing, the AAR and EER parameters are obtained from each trial whose output is further fed to the feature selector and classifier to yield the final output, as discussed in the following section.

\section{RESULTS AND DISCUSSION}

After feature extraction, we have a data-set associated with AAR features and another data-set corresponding to the EER features. Fig. 5a shows the AAR features for the sub-classes of the wrist movements corresponding to an EEG signal of 3seconds from FC5 electrode. Fig. 5b demonstrates the EER features for the sub-classes of the finger movements obtained from an EEG signal of 3 seconds from the electrodes considered in the order stated in the previous section. Following the extraction of features, Sequential Forward Selection selects a subset of the relevant features from the original dataset, which is mentioned in Table I.
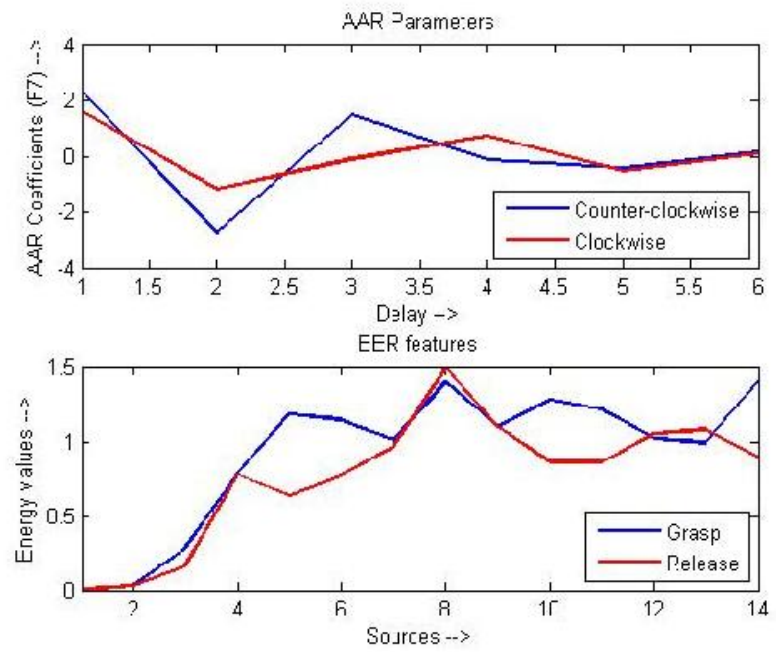

Fig. 4. Plot of AAR parameters and EER features 
TABLE I. Index of Selected Features Using Sequential Forward FEATURE SELECTION

\begin{tabular}{|l|l|l|}
\hline Classifiers & \multicolumn{1}{|c|}{ Selected AAR features } & Selected EER features \\
\hline DLRT & $62,64,10,45,63,56,16,2,39,27$ & $2,14,6,4,11,3,7,12,5,13$ \\
\hline FLD & $14,11,61,8,79,30,31,6,5,4$ & $9,11,5,1,6,13,8,2,3,4$ \\
\hline Nä̈ve Bayes & $14,84,11,24,6,1,4,78,5,37$ & $11,10,3,9,12,14,1,13,2,5$ \\
\hline SVM & $63,60,42,36,12,24,66,61,54,72$ & $2,6,10,11,5,1,7,4,12,8$ \\
\hline
\end{tabular}

The classification results implementing the scheme mentioned in Fig. 1 are tabulated in Table II when AAR parameters are used as features. Similarly, Table III specifies the results corresponding to features extracted with EER criterion. The average classification accuracy and the average execution time of the total scheme over a large number of dataset are considered as performance metrics. Also, the minimum accuracy and the maximum accuracy with any classifier is noted which indicates the variance of the results. The overall execution time includes the time for processing, feature extraction, feature selection and classifying the featureset of 200 observations using any of the stated classifiers.

TABLE II. Classification Results with AAR Parameters as FEATURES

\begin{tabular}{|l|l|c|c|c|c|}
\hline \multirow{2}{*}{$\begin{array}{c}\text { Classification Accuracy } \\
\text { \& Computation Time }\end{array}$} & \multicolumn{4}{|c|}{ Classifiers } \\
\cline { 2 - 6 } & Min (\%) & 60.00 & 50.00 & 71.67 & 72.50 \\
\cline { 2 - 6 } & Max (\%) & 90.00 & 100.00 & 90.00 & 95.00 \\
\cline { 2 - 6 } & Mean (\%) & 74.40 & 78.57 & 78.63 & 86.90 \\
\cline { 2 - 6 } $\begin{array}{l}\text { So Feature } \\
\text { Selection }\end{array}$ & Avg. & 51.6620 & 50.8401 & 54.9971 & 51. \\
& Time (s) & & & & \\
& Min (\%) & 81.67 & 76.67 & 81.67 & 80.00 \\
\hline \multirow{4}{*}{$\begin{array}{l}\text { Sequential } \\
\text { Forward } \\
\text { Selection }\end{array}$} & Max (\%) & 97.50 & 100.00 & 95.00 & 100.00 \\
\cline { 2 - 6 } & Mean (\%) & 88.69 & 87.62 & 88.51 & 95.72 \\
\cline { 2 - 6 } & Avg. & 103. & 132. & 206.5524 & 108. \\
& Time (s) & 0023 & 0931 & 8799 \\
\hline
\end{tabular}

TABLE III. Classification Results With FeAtures BASED on EER CRITERION

\begin{tabular}{|l|l|c|c|c|c|}
\hline \multirow{2}{*}{$\begin{array}{c}\text { Classification Accuracy } \\
\text { \& Computation Time }\end{array}$} & \multicolumn{5}{|c|}{ Classifiers } \\
\cline { 2 - 6 } & Min (\%) & 70.00 & 66.67 & 60.00 & 70.00 \\
\hline \multirow{4}{*}{$\begin{array}{l}\text { No Feature } \\
\text { Selection }\end{array}$} & Max (\%) & 85.00 & 92.50 & 83.33 & 100.00 \\
\cline { 2 - 6 } & Mean (\%) & 74.94 & 81.49 & 72.80 & 87.98 \\
\cline { 2 - 6 } & $\begin{array}{l}\text { Avg. } \\
\text { Time (s) }\end{array}$ & 2.1477 & 2.2620 & 2.4806 & 2.2516 \\
\hline \multirow{3}{*}{$\begin{array}{l}\text { Sequential } \\
\text { Forward } \\
\text { Selection }\end{array}$} & Min (\%) & 70.00 & 70.00 & 65.00 & 75.00 \\
\cline { 2 - 6 } & Max (\%) & 88.33 & 92.50 & 87.50 & 100 \\
\cline { 2 - 6 } & Mean (\%) & 80.77 & 82.44 & 77.44 & 90.24 \\
\cline { 2 - 6 } & $\begin{array}{l}\text { Avg. } \\
\text { Time (s) }\end{array}$ & 7.3266 & 7.6746 & 11.6486 & 8.2449 \\
\hline
\end{tabular}

From the above results, it can be concluded that although the AAR features provide very high recognition rate as compared to EER features, it takes up a considerable amount of time which is not suitable for real-time application of the work. The EER features, on the other hand, provide significant classification accuracies within a short time. The classification accuracy is more when the feature selection step is used. Among all the classifiers considered, SVM outperforms others when accuracy is considered. So, for the purpose of offline classification this study suggests the use of AAR features extracted from the EEG data. However, integration of the scheme with robots presents a real-time scenario for which the study recommends the combination of EER features extracted from EEG, selected by sequential forward selection and classified using SVM.

A comparison of mean accuracy obtained by the methods used by previous researchers is provided in Table IV. We note that our proposed method yields highest overall accuracy as compared to the other techniques.

TABLE IV. COMPARISON WITH PREVIOUS WORK

\begin{tabular}{|l|c|}
\hline \multicolumn{1}{|c|}{ Methods } & $\begin{array}{c}\text { Mean } \\
\text { Accuracy } \\
\text { (\%) }\end{array}$ \\
\hline $\begin{array}{l}\text { Classification (wavelet coefficients, PSD estimate, band power } \\
\text { estimate-kNN) [9] }\end{array}$ & 75.00 \\
\hline Performance Analysis (band power estimate-kNN) [10] & 84.29 \\
\hline Classification (mean Power- spatial feature selection-LDA) [11] & 82.69 \\
\hline \begin{tabular}{l|l} 
Classification (wavelet transform, AR coefficients-LDA) [12] \\
Two-fold classification (wavelet coefficients/power spectral \\
estimate-RBF-SVM) [13]
\end{tabular} & 90.00 \\
\hline $\begin{array}{l}\text { Intelligent Algorithms (wavelet coefficients, PSD, average } \\
\text { power-PCA-RBF-SVM) [14] }\end{array}$ & 82.14 \\
\hline Proposed Method (EER-SFS-SVM) & 90.24 \\
\hline (AAR-SFS-SVM) & 95.72 \\
\hline
\end{tabular}

\section{CONCLUSION AND FUTURE DIRECTION}

The work aims at selecting a suitable feature and classifier that generates outcome trading off with the demands of favorable recognition rate and small computation time for online use of the two-level classification of wrist and finger based motor imagery signals. The average AAR coefficients and EER based features are extracted from the processed EEG signals, which is classified by the four classifiers: DLRT, FLD Naïve Bayes, SVM, both before and after a Sequential Forward Selection is implemented. From the noted classification result, we find features extracted from EER criterion when selected by sequential forward search to find a subset of 10 features yield a desirable mean accuracy of $90.24 \%$ within a short-period of time i.e., in 8.2449 seconds. In online mode, the feature-set will be much smaller and hence, the time required reduces further to a lesser duration.

In our future work, we aim to apply this method to control a robot hand in a real-time scenario. This can further assist as a rehabilitative tool to increase the functionality of a disabled person. 


\section{ACKNOWLEDGMENT}

This study has been supported by University Grants Commission (UGC), India; University with Potential for Excellence Program (UGC-UPE) (Phase II) in Cognitive Science, Jadavpur University and Council of Scientific and Industrial Research (CSIR), India.

\section{REFERENCES}

[1] Schwartz A.B., Cui X.T., Weber D.J., Moran D.W. "Brain Controlled Interfaces: Movement Restoration using Neural Prosthetics." Neuron 52, October 2006, pp. 205-220.

[2] Lebedev M.A., Nicoleis, "Brain-machine interface: Past, present and future” , Trends Neurosci. 29(9), September 2006, pp. 536-546.

[3] McFarland Dennis J., Wolpaw Jonathan R. "Brain-Computer Interface Operation of Robotic and Prosthetic Devices", IEEE Computer Society, 2008.

[4] Daly Janis J, Wolpaw Jonathan R , "Brain-computer interfaces in neurological rehabilitation", Lancet Neurol 2008; 7: pp. 103243

[5] Anderson R.A., Musallam S., Pesaran B., "Selecting the signals for a brain-machine interface", Curr Opin Neurobiol Vol.14 (6), December 2004, pp.720-726.

[6] Besio, W., Koka, K., Patwardhan, R. (2004, September). "Computer simulation and tank experimental verification of concentric ring electrodes". In Engineering in Medicine and Biology Society, 2004. IEMBS'04. 26th Annual International Conference of the IEEE (Vol. 1, pp. 2243-2246). IEEE.

[7] Matsunanga T., Katayama Y., Hayami T., Iramina K. "Measurement of mu/beta ERD and gamma ERS during the imagination of body parts movement." $30^{\text {th }}$ Annual International IEEE EMBS Conference Vancouver Canada, August 2008.

[8] Hema C.R., Paulraj M.P., Yaacob S., Adom A.H., Nagarajan R. "Recognition of motor imagery of hand movements for a BMI using PCA features." International Conference on Electronic Design, Penang, Malaysia, December 2008.

[9] Khasnobish, A., Bhattacharyya, S., Konar, A., Tibarewala, D. N., "K-Nearest neighbor classification of left-right limb movement using EEG data". In oral presentation in International conference on Biomedical Engineering and assistive technologies, NIT Jalandhar 2010.

[10] Bhattacharyya, S., Khasnobish, A., Chatterjee, S., Konar, A., Tibarewala, D. N., "Performance analysis of LDA, QDA and KNN algorithms in left-right limb movement classification from EEG data". In Systems in Medicine and Biology (ICSMB), December 2010 International Conference on (pp. 126-131). IEEE.

[11] Caracillo, R. C., Castro, M. C. F. (2013, February), "Classification of executed upper limb movements by means of EEG”. In Biosignals and Biorobotics Conference (BRC), 2013 ISSNIP (pp. 1-6). IEEE.

[12] Xu, B., Song, A., Wu, J. (2007, July), "Algorithm of imagined left-right hand movement classification based on wavelet transform and AR parameter model". In Bioinformatics and
Biomedical Engineering, 2007. ICBBE 2007. The 1st International Conference on (pp. 539-542). IEEE.

[13] Khasnobish, A., Bhattacharyya, S., Konar, A., Tibarewala, D. N., Nagar, A. K., "A Two-fold classification for composite decision about localized arm movement from EEG by SVM and QDA techniques". In Neural Networks (IJCNN), July 2011, International Joint Conference on (pp. 1344-1351). IEEE.

[14] Bhattacharyya, S., Khasnobish, A., Konar, A., Tibarewala, D. N., Nagar, A. K., "Performance analysis of left/right hand movement classification from EEG signal by intelligent algorithms". In Computational Intelligence, Cognitive Algorithms, Mind, and Brain (CCMB), April 2011 IEEE Symposium on (pp. 1-8). IEEE.

[15] Schlögl, A., Lee, F., Bischof, H., Pfurtscheller, G., "Characterization of four-class motor imagery EEG data for the BCI-competition 2005". Journal of neural engineering, 2(4), L14.

[16] Schlögl, A., Lugger, K., Pfurtscheller, G. (1997, November), "Using adaptive autoregressive parameters for a brain-computerinterface experiment". In Engineering in Medicine and Biology Society, 1997. Proceedings of the 19th Annual International Conference of the IEEE (Vol. 4, pp. 1533-1535). IEEE.

[17] Sun, S., "The extreme energy ratio criterion for EEG feature extraction". In Artificial Neural Networks-ICANN 2008 (pp. 919-928). Springer Berlin Heidelberg.

[18] Tu, W., Sun, S. (2011, July), "Semi-supervised feature extraction with local temporal regularization for EEG Classification". In Neural Networks (IJCNN), The 2011 International Joint Conference on (pp. 75-80). IEEE.

[19] Marcano-Cedeño, A., Quintanilla-Domínguez, J., CortinaJanuchs, M. G., Andina, D. (2010, November), "Feature selection using Sequential Forward Selection and classification applying Artificial Metaplasticity Neural Network". In IECON 2010-36th Annual Conference on IEEE Industrial Electronics Society (pp. 2845-2850). IEEE.

[20] Wang, L., Shen, C., Hartley, R. (2011, December), "On the optimality of sequential forward feature selection using class separability measure". In Digital Image Computing Techniques and Applications (DICTA), 2011 International Conference on (pp. 203-208). IEEE.

[21] Alexandre-Cortizo, E., Rosa-Zurera, M., Lopez-Ferreras, F. (2005, November). Application of fisher linear discriminant analysis to speech/music classification. In Computer as a Tool, 2005. EUROCON 2005. The International Conference on (Vol. 2, pp. 1666-1669). IEEE.

[22] Remus, J.J.; Morton, K.D.; Torrione, P.A.; Tantum, S.L.; Collins, L.M., "Comparison of a distance-based likelihood ratio test and k-nearest neighbor classification methods," Machine Learning for Signal Processing, 2008. MLSP 2008. IEEE Workshop on , vol., no., pp.362,367, 16-19 Oct. 2008.

[23] Shenoi, K., Agrawal, B. (1982), "A design algorithm for constrained equiripple digital filters". Acoustics, Speech and Signal Processing, IEEE Transactions on, 30(2), 206-211. 